\title{
Dinâmica de herbicidas aplicados em pré-emergência sobre palha de cana-de- açúcar em diferentes regimes hídricos ${ }^{1}$
}

\author{
Dynamic of herbicides applied in preemergence on sugarcane straw under different \\ water regimes
}

Ana Beatriz Campos Almeida Prado ${ }^{2}$; Flávio Eduardo Botelhos Obara²; Caio Augusto Grossi Brunharo $^{2}$; Marcel Sereguin Cabral Melo ${ }^{2}$; Pedro Jacob Christoffoleti ${ }^{3}$; Marcelo Corrêa Alves ${ }^{4}$

Resumo - A manutenção da cobertura morta sobre o solo pode reduzir o potencial de infestação de plantas daninhas, assim como pode afetar a transposição dos herbicidas, a dinâmica de molhamento e a lavagem da palha pela água das chuvas. O trabalho teve como objetivos avaliar a dinâmica dos herbicidas diuron, metribuzin e tebuthiuron em diferentes regimes hídricos com e sem palha, e apontar as principais relações entre a presença ou ausência de palha na remoção dos herbicidas pela água da chuva e influência na eficácia de controle de plantas daninhas. $\mathrm{O}$ delineamento experimental foi o inteiramente casualizado em esquema fatorial $6 \times 3 \times 2$, com quatro repetições, onde foram testados seis níveis para o fator chuva simulada (testemunha, 0,5 , 10,20 e $20+20 \mathrm{~mm}$ ), três herbicidas (diuron a $2,0 \mathrm{~kg} \mathrm{ha}^{-1}$; metribuzin a $1,92 \mathrm{~kg} \mathrm{ha}^{-1} \mathrm{e}$ tebuthiuron a $\left.1,2 \mathrm{~kg} \mathrm{ha}^{-1}\right)$ e duas quantidades de palha $\left(0\right.$ e $\left.10 \mathrm{t} \mathrm{ha}^{-1}\right)$. Um dia após a aplicação dos herbicidas a palha foi removida dos vasos e as espécies Ipomoea grandifolia e Cucumis sativus foram semeadas, avaliando-se o controle aos 15 e 30 dias após a aplicação e 10 e 21 dias após a aplicação, respectivamente. $\mathrm{O}$ herbicida diuron foi menos eficaz nos tratamentos com palha emPcomparação ao metribuzin e tebuthiuron. A palha afetou de maneira significativa o controle das espécies I. grandifolia e $C$. sativus pelos herbicidas na ausência de chuva simulada. Chuva de $20 \mathrm{~mm}$ foi suficiente para remover os herbicidas da palha para o solo e promover níveis adequados de controle das plantas daninhas.

Palavras-chaves: Irrigação, controle, diuron, metribuzin, tebuthiuron

\begin{abstract}
Maintenance of mulch on the soil surface can reduce the potential for weed infestations, and may affect transposition of the herbicides, the dynamics of wetting and washing the straw by rainwater. The study had as objectives evaluate the dynamics of the herbicides diuron, tebuthiuron and metribuzin in different water regimes with and without straw, and point out the main relationships between the presence or absence of straw removal of herbicides by rainwater and influence on the effectiveness of weed control. The experimental design was completely randomized in factorial $6 \times 3 \times 2$, with four replications, being tested six levels of the rain factor (control, 0, 5, 10, 20 and $20+20 \mathrm{~mm}$ ), three herbicides (diuron $2000 \mathrm{~g} \mathrm{~h}^{-1}$ ),

\footnotetext{
${ }^{1}$ Recebido para publicação em 08/05/2013 e aceito em 31/12/2013.

${ }^{2}$ Aluno do programa de pós-graduação do programa de Fitotecnia da ESALQ/USP, Av. Pádua Dias, 11, 13418-900, Piracicaba, SP. E-mail:<abeatriz.prado@gmail.com>.(Autor para correspondência).

${ }^{3}$ Professor associado do Departamento de Produção Vegetal da ESALQ/USP

${ }^{4}$ Membro da Seção Técnica de Informática (ESALQ/USP)
} 
metribuzin (1920 $\left.\mathrm{g} \mathrm{ha}^{-1}\right)$ and tebuthiuron $\left(1200 \mathrm{~g} \mathrm{ha}^{-1}\right)$, and two quantities of straw (0 and $10 \mathrm{t} \mathrm{ha}^{-}$ ${ }^{1}$ ). One day after application, straw was removed from the pots and Ipomoea grandifolia and Cucumis sativus species were sown in pots, evaluating the control at 15 and 30 and 10 and 21 days after application, respectively. Diuron was less effective in the treatments with straw compared to tebuthiuron and metribuzin. The straw significantly affected the weed control by herbicides in the absence of rain, and a rain of $20 \mathrm{~mm}$ was sufficient to cause leaching of the herbicides into the soil, making them available for absorption by the plants and to promote excellent levels of control thereof.

Keywords: Irrigation, control, diuron, metribuzin, tebuthiuron

\section{Introdução}

A camada de palha depositada na superfície do solo, resultante da colheita mecanizada da cana-de-açúcar é uma das barreiras para o uso de herbicidas com ação exclusiva ou preferencial no solo (Negrisoli et al, 2007). A manutenção da cobertura morta sobre a superfície do solo pode reduzir o potencial de infestação de plantas daninhas, tanto pela limitação da variação da temperatura na superfície do solo como pela formação de uma barreira física a ser transposta pela planta daninha em germinação (Rossi et al., 2013). E ainda pode dificultar o desempenho dos herbicidas. Todos esses fatores estão relacionados à transposição do herbicida pela palha, à dinâmica de molhamento e lavagem da palha pela água das chuvas (Maciel \& Velini, 2005).

No entanto, algumas espécies do gênero Ipomoea adaptaram-se a essa mudança germinando mesmo na presença de palha. De acordo com Correia \& Durigan (2004), a presença de palha ocasionou redução na densidade populacional das espécies Brachiaria decumbens, Sida spinosa e Digitaria horizontalis. Por outro lado, o mesmo não foi observado para I. grandifolia, I. hederifolia e I. quamoclit. As espécies de plantas daninhas pertencentes aos gêneros Ipomoea e Merremia, podem causar sérios danos à cultura da cana-de-açúcar, principalmente em áreas de colheita mecanizada (Alves, 2010).

Lamoreaux et al. (1993) ressaltaram que o transporte de herbicidas da palha para o solo depende de vários fatores, como as características físico-químicas inerentes a cada herbicida, a capacidade da palha de cobrir o solo e de reter o herbicida e o período em que a área permanece sem chuva após a aplicação. A chuva é responsável pelo transporte do herbicida até o solo. Diversos trabalhos demonstraram que os primeiros $20 \mathrm{~mm}$ iniciais são responsáveis pelo carregamento dos herbicidas, apresentando alta capacidade de transporte dos mesmos (Velini et al., 2003; Rossi et al., 2013; Tofoli et al., 2009).

Os herbicidas diuron, metribuzin e tebuthiuron inibem o Fotossistema II, mas, possuem características físicas e químicas distintas que podem fazer com que sua dinâmica e eficácia para as plantas daninhas sejam diferentes quando aplicados sobre palha de cana-de-açúcar. Os herbicidas apresentam comportamentos diferentes com relação à eficácia de controle sobre as plantas daninhas, sendo que diuron por apresentar menor solubilidade em água (42 $\mathrm{mg} \mathrm{L}^{-1}$ a $\left.25^{\circ} \mathrm{C}\right)$ necessita de maior umidade para ser removido da palha e assim atingir seu efeito de controle sobre as plantas daninhas, diferentemente de metribuzin e tebuthiuron cuja solubilidade em água é $1.100 \mathrm{mg} \mathrm{L}^{-1}$ a $20^{\circ} \mathrm{C}$ e $2.570 \mathrm{mg} \mathrm{L}^{-1}$ a $20^{\circ} \mathrm{C}$. Portanto, o trabalho teve como objetivo conhecer a dinâmica dos herbicidas diuron, metribuzin e tebuthiuron em diferentes regimes hídricos com e sem palha, apontando a principal relação que a presença ou ausência de palha tem na remoção dos herbicidas pela água da chuva e relacionar esses efeitos com à eficácia de controle das plantas daninhas. 


\section{Material e Métodos}

$\mathrm{O}$ experimento foi realizado em condições de casa-de-vegetação do Departamento de Produção Vegetal da Escola Superior de Agricultura Luiz de Queiroz, Piracicaba/SP, durante os meses de Fevereiro e Março de 2013.

$\mathrm{O}$ delineamento experimental foi inteiramente casualizado em esquema fatorial 6 x 3 x 2, com quatro repetições, em vasos de capacidade de 1 litro. Foram testados seis níveis para o fator simulação de chuva (testemunha, 0, 5, 10, 20 e $20+20 \mathrm{~mm}$ ), três herbicidas (diuron a 2,0 $\mathrm{kg} \mathrm{ha}^{-1}$; metribuzin a $1,92 \mathrm{~kg} \mathrm{ha}^{-1}$ e tebuthiuron a $1,2 \mathrm{~kg} \mathrm{ha}^{-1}$ ) e duas quantidades de palha da variedade RB86-7515 $\left(0\right.$ e $\left.10 \mathrm{t} \mathrm{ha}^{-1}\right)$. A irrigação dos vasos nos diferentes volumes descritos foi realizada logo após a aplicação dos herbicidas. No volume 20 $+20 \mathrm{~mm}$, foi simulada uma chuva de $20 \mathrm{~mm}$ logo após a aplicação dos herbicidas e mais uma chuva de $20 \mathrm{~mm}$ um dia após a aplicação.

As doses dos herbicidas utilizados foram consideradas em função da textura do solo, seguindo as recomendações de Rodrigues \& Almeida (2011). As análises químicas e físicas do solo foram realizadas no Laboratório de Solos, do Departamento de Ciência do Solo da ESALQ/USP, e encontram-se representadas na Tabela 1.

Tabela 1. Propriedades químicas e físicas do solo utilizado no experimento. Piracicaba/SP, 2013.

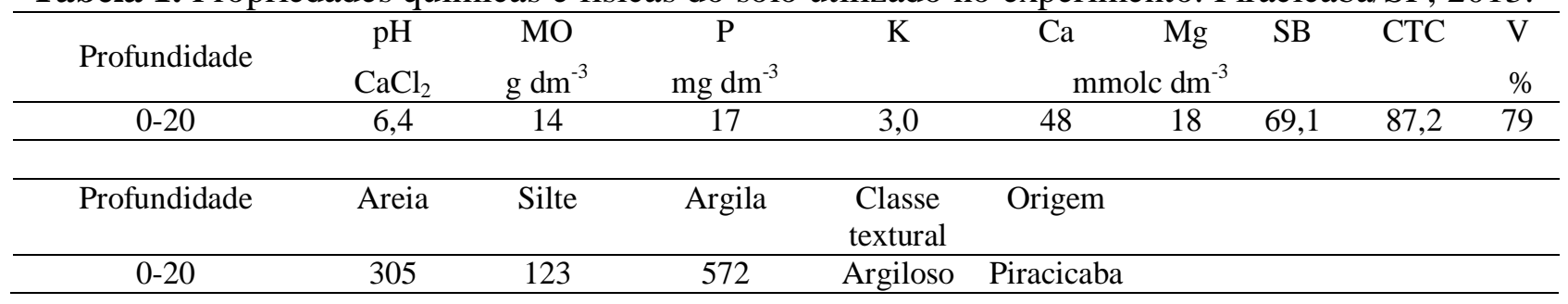

Os herbicidas foram aplicados com o auxílio de pulverizador costal pressurizado por $\mathrm{CO}_{2}$, acoplado a uma barra de pulverização com uma ponta jato plano DG 11002, que proporcionou a aplicação de $200 \mathrm{~L} \mathrm{ha}^{-1}$ de calda. Após a aplicação dos herbicidas, realizaram-se as simulações de chuva (testemunha, 0, 5, 10, 20 e $20+20 \mathrm{~mm}$ ) para cada tratamento com e sem palha.

Após a secagem total da palha, retirouse a mesma do vaso. Um dia após a aplicação foram semeadas as espécies Cucumis sativus e Ipomoea grandifolia, na quantidade de 5 e 8 sementes por vaso, respectivamente. Os vasos foram diariamente submetidos à irrigação por aspersão, para emergência e desenvolvimento das plantas.

Aos 10 e 21 dias após aplicação (DAA) realizaram-se avaliações visuais de controle para a espécie Cucumis sativus por meio de escala percentual de notas, em que zero correspondeu a nenhuma injúria na planta e 100, à morte da planta, seguindo-se metodologia proposta pela ALAM (1974). Aos 21 DAA, a parte aérea das plantas foram coletadas e levadas para secagem em estufa de circulação forçada por 72 horas, a temperatura constante de $60^{\circ} \mathrm{C}$, para quantificação da matéria seca. $\mathrm{O}$ mesmo procedimento foi realizado para a espécie Ipomoea grandifolia. No entanto, as avaliações visuais de controle foram realizadas aos 15 e 30 DAA, e a coleta da parte aérea das plantas aos 30 DAA. Apenas os dados das avaliações de 30 dias foram submetidos a análise estatística.

Os dados obtidos foram submetidos à análise de variância e efeitos significativos, ao teste de Tukey com nível de significância de $5 \%$, por meio do sistema SAS (SAS Institute Inc. The SAS System release 9.3. SAS Institute Inc., Cary:NC, 2010). Optou-se por utilizar como unidade de dispersão o desvio padrão por 
permitir melhor visualização dos dados.

\section{Resultados e Discussão}

O herbicida diuron resultou em baixo nível de controle de I. grandifolia nos menores volumes de precipitação (0, 5 e $10 \mathrm{~mm}$ ), quando comparado aos demais produtos. No entanto, após chuva simulada de $20 \mathrm{~mm}$ os herbicidas não apresentaram diferenças significativas no controle dessa espécie, indicando que a precipitação de $20 \mathrm{~mm}$ foi suficiente para que os herbicidas fossem removidos da palha atingindo o solo.

Metribuzin apresentou desempenho razoável mesmo nas precipitações de 5 e 10 $\mathrm{mm}$. Para o herbicida tebuthiuron foram obtidos ótimos resultados de controle, acima de 94\% quando foi simulada chuva de apenas 5 mm logo após a aplicação.

Metribuzin e tebuthiuron por serem de maior solubilidade exigem menor quantidade de água para serem extraídos da palha, liberados no solo e portanto disponíveis para absorção das plantas.

Tabela 2. Valores médios para avaliação de controle (\%) de I. grandifolia aos 30 dias após a aplicação (DAA) para diuron, metribuzin e tebuthiuron em diferentes precipitações após a aplicação na presença e ausência de palha de cana-de-açúcar. (SD = Desvio Padrão). Piracicaba/SP, 2013.

\begin{tabular}{cccc}
\hline $\begin{array}{c}\text { Precipitação } \\
\left(\mathbf{m m} \mathbf{~ h a}^{-1}\right)\end{array}$ & Diuron \pm SE & Metribuzin \pm SE & Tebuthiuron \pm SE \\
\cline { 2 - 4 } $\mathbf{0}$ & $41,88 \pm 42,11 \mathrm{Db}$ & $66,25 \pm 44,25 \mathrm{Ca}$ & $64,94 \pm 39,73 \mathrm{Ba}$ \\
$\mathbf{5}$ & $68,94 \pm 22,12 \mathrm{Cb}$ & $78,75 \pm 21,41 \mathrm{BCab}$ & $94,25 \pm 13,22 \mathrm{Aa}$ \\
$\mathbf{1 0}$ & $74,00 \pm 35,69 \mathrm{BCb}$ & $89,00 \pm 20,31 \mathrm{ABa}$ & $99,88 \pm 0,34 \mathrm{Aa}$ \\
$\mathbf{2 0}$ & $83,88 \pm 23,90 \mathrm{ABa}$ & $97,94 \pm 4,36 \mathrm{ABa}$ & $99,94 \pm 0,25 \mathrm{Aa}$ \\
$\mathbf{2 0 + 2 0}$ & $96,69 \pm 9,92 \mathrm{Aa}$ & $99,38 \pm 2,50 \mathrm{Aa}$ & $100,00 \pm 0,00 \mathrm{Aa}$ \\
Testemunha & $0,00 \pm 0,00 \mathrm{Ea}$ & $0,00 \pm 0,00 \mathrm{Da}$ & $0,00 \pm 0,00 \mathrm{Ca}$ \\
\hline
\end{tabular}

Médias seguidas por letras iguais, maiúsculas nas colunas e minúsculas nas linhas, não diferem entre si pelo teste de Tukey nível de significância de 5\%.

A presença de $10 \mathrm{t}$ de palha por hectare diferiu significativamente no controle de $I$. grandifolia para os três herbicidas, observando-se menores médias de controle nos tratamentos com a presença de palha, o que indica que a mesma afetou a movimentação dos herbicidas para o solo (Tabela 3). Ao comparar os herbicidas na presença de palha, observou-se que o diuron teve a eficácia de controle mais afetada devido a sua maior dificuldade de remoção da palha do que os outros herbicidas, os quais apresentaram médias de controle significativamente superiores. Resultados semelhantes foram obtidos por Cavenaghi et al. (2002), os quais ao avaliarem a dinâmica de diuron em palha de cana-de-açúcar verificaram redução significativa na transposição do herbicida em quantidades de palha maiores que $2 \mathrm{t} \mathrm{ha}^{-1}$, sendo que para quantidade de palha superior a $15 \mathrm{t} \mathrm{ha}^{-1}$, a transposição de diuron foi nula. No entanto, os autores também constataram que, após a simulação de uma chuva de $50 \mathrm{~mm}$, houve expressivo aumento na transposição do diuron pela palha, chegando a $65 \%$ de remoção. Monquero et al. (2009), ao estudarem a eficácia de herbicidas no controle de $I$. grandifolia em diferentes quantidades de palha, concluíram que com $10 \mathrm{t} \mathrm{ha}^{-1}$ de palha houve diferença entre os produtos, e que a mistura diuron+ hexazinone resultou no pior nível de controle $(22,5 \%)$. 
Tabela 3. Valores médios para avaliação de controle (\%) de I. grandifolia aos 30 dias após aplicação (DAA) para diuron, metribuzin e tebuthiuron em diferentes precipitações após a aplicação na presença e ausência de palha de cana-de-açúcar. (SD = Desvio Padrão). Piracicaba/SP, 2013.

\begin{tabular}{cccc}
\hline \multirow{2}{*}{ Palha } & \multicolumn{3}{c}{ Herbicidas } \\
\cline { 2 - 4 } & Diuron \pm SE & Metribuzin \pm SE & Tebuthiuron \pm SE \\
\hline Com & $47,29 \pm 40,31 \mathrm{Bb}$ & $63,17 \pm 41,89 \mathrm{Ba}$ & $70,15 \pm 42,17 \mathrm{Ba}$ \\
Sem & $74,50 \pm 37,70 \mathrm{Aa}$ & $80,60 \pm 37,09 \mathrm{Aa}$ & $82,85 \pm 37,49 \mathrm{Aa}$ \\
\hline
\end{tabular}

Médias seguidas por letras iguais, maiúsculas nas colunas e minúsculas nas linhas, não diferem entre si pelo teste de Tukey nível de significância de 5\%.

O controle de I. grandifolia quando houve a presença da palha no momento da aplicação foi significativamente diferente nas precipitações simuladas de chuva de 0,5 e 10 $\mathrm{mm}$, quando comparado aos tratamentos em que os herbicidas foram aplicados apenas sobre o solo (Tabela 4). A partir da precipitação de $20 \mathrm{~mm}$ não foram observadas diferenças significativas no controle da referida espécie. Assim, o efeito observado foi da retenção do herbicida pela palha e da sua capacidade de transpô-la nos diferentes volumes de chuva.

Os resultados obtidos concordam com Negrisoli et al. (2007) que observaram excelente controle de I. grandifolia pelo tebuthiuron aplicado sobre a palha de cana-deaçúcar, quando ocorreu precipitação de $20 \mathrm{~mm}$, um dia após a sua aplicação. Negrisoli et al. (2009) também verificaram bons níveis de controle de I. grandifolia quando aplicou-se oxyfluorfen sobre $5 \mathrm{t} \mathrm{ha}^{-1}$ de palha de cana-deaçúcar, seguido pela simulação de $2,5 \mathrm{~mm}$ de chuva. Os tratamentos com palha apresentaram diferenças significativas nas precipitações 20 $\mathrm{mm}$ e $20+20 \mathrm{~mm}$, em que as porcentagens médias de controle das plantas daninhas foram superiores quando comparadas aos mesmos valores para 0,5 e $10 \mathrm{~mm}$. Resultados similares foram obtidos por Tofoli et al. (2009), que ao avaliar a dinâmica de tebuthiuron em palha de cana-de-açúcar, relataram que os primeiros $20 \mathrm{~mm}$ de chuva foram fundamentais para $\mathrm{o}$ processo de carregamento do herbicida para o solo, independentemente da quantidade de palha, onde após $20 \mathrm{~mm}$ houve tendência dos dados serem similares. Rossi et al. (2013) constataram que a extração de $99 \%$ do metribuzin aplicado sobre $10 \mathrm{t} \mathrm{ha}^{-1}$ somente foi obtida com simulação de $21,5 \mathrm{~mm}$ de chuva. Cavenaghi et al. (2007) concluíram que $75 \%$ do amicarbazone aplicado foi removido por uma lâmina de $20 \mathrm{~mm}$ de chuva. Esses dados indicam que $20 \mathrm{~mm}$ de chuva são suficientes para que os herbicidas estudados sejam removidos da palha e que o aumento desse volume de chuva $(20 \mathrm{~mm})$ não resultará em maior remoção do produto na mesma proporção.

Ao analisar a matéria seca de $I$. grandifolia observou-se que as diferenças entre as precipitações foram menos evidentes não apresentando diferenças significativas entre os herbicidas (Tabela 5). No entanto, ao comparar as precipitações para cada herbicida, concluiuse que o diuron, sem a simulação de chuva após a aplicação, resultou em matéria seca semelhante à testemunha sem herbicida.

Portanto, que o mesmo não foi eficaz no controle da planta daninha na ausência de precipitação. As diferentes precipitações não afetaram a eficácia do metribuzin quando comparadas entre si, diferenciando-se apenas da testemunha. Para tebuthiuron foram encontradas diferenças entre a testemunha e as precipitações de 5, 10, 20 e 20+20 mm. 
Tabela 4. Valores médios para avaliação de controle (\%) de I. grandifolia aos 30 dias após a aplicação (DAA) em diferentes precipitações após o tratamento com e sem palha utilizando os herbicidas diuron, metribuzin e tebuthiuron (SD = Desvio Padrão). Piracicaba/SP, 2013.

\begin{tabular}{ccc}
\hline \multirow{2}{*}{ Precipitação $\left(\mathbf{m m ~ h a}^{-\mathbf{1}}\right)$} & Com \pm SE & Palha \\
\cline { 2 - 3 } & $24,17 \pm 31,75 \mathrm{Db}$ & $91,21 \pm 19,28 \mathrm{Aa}$ \\
$\mathbf{0}$ & $70,42 \pm 22,69 \mathrm{Cb}$ & $90,88 \pm 15,01 \mathrm{Aa}$ \\
$\mathbf{1 0}$ & $79,71 \pm 33,20 \mathrm{BCb}$ & $95,54 \pm 10,11 \mathrm{Aa}$ \\
$\mathbf{2 0}$ & $89,54 \pm 20,86 \mathrm{ABa}$ & $98,29 \pm 4,07 \mathrm{Aa}$ \\
$\mathbf{2 0 + 2 0}$ & $97,38 \pm 8,31 \mathrm{Aa}$ & $100,00 \pm 0,00 \mathrm{Aa}$ \\
Testemunha & $0,00 \pm 0,00 \mathrm{Ea}$ & $0,00 \pm 0,00 \mathrm{Ba}$ \\
\hline
\end{tabular}

Médias seguidas por letras iguais, maiúsculas nas colunas e minúsculas nas linhas, não diferem entre si pelo teste de Tukey nível de significância de 5\%.

Tabela 5. Valores médios de matéria seca (g) da parte aérea de Ipomoea grandifolia aos 30 dias após a aplicação (DAA) para diuron, metribuzin e tebuthiuron em diferentes precipitações na presença e ausência de palha (SD = Desvio Padrão). Piracicaba/SP, 2013.

\begin{tabular}{cccc}
\hline $\begin{array}{c}\text { Precipitação } \\
\left(\mathbf{m m ~ h a}^{-1}\right)\end{array}$ & Diuron \pm SE & Metribuzin \pm SE & Tebuthiuron \pm SE \\
\cline { 2 - 4 } & $0,25 \pm 0,28 \mathrm{Aba}$ & $0,26 \pm 0,45 \mathrm{Ba}$ & $0,07 \pm 0,11 \mathrm{Aba}$ \\
$\mathbf{5}$ & $0,10 \pm 0,14 \mathrm{Ba}$ & $0,13 \pm 0,16 \mathrm{Ba}$ & $0,00 \pm 0,00 \mathrm{Ba}$ \\
$\mathbf{1 0}$ & $0,10 \pm 0,21 \mathrm{Ba}$ & $0,07 \pm 0,14 \mathrm{Ba}$ & $0,00 \pm 0,00 \mathrm{Ba}$ \\
$\mathbf{2 0}$ & $0,07 \pm 0,09 \mathrm{BCa}$ & $0,01 \pm 0,01 \mathrm{Ba}$ & $0,00 \pm 0,00 \mathrm{Ba}$ \\
$\mathbf{2 0 + 2 0}$ & $0,03 \pm 0,06 \mathrm{Ca}$ & $0,01 \pm 0,02 \mathrm{Ba}$ & $0,00 \pm 0,00 \mathrm{Ba}$ \\
Testemunha & $0,42 \pm 0,32 \mathrm{Ab}$ & $0,80 \pm 0,40 \mathrm{Aa}$ & $0,33 \pm 0,23 \mathrm{Ab}$ \\
\hline
\end{tabular}

Médias seguidas por letras iguais, maiúsculas nas colunas e minúsculas nas linhas, não diferem entre si pelo teste de Tukey nível de significância de 5\%.

Em relação ás médias de matéria seca na presença ou ausência de palha (Tabela 6), notou-se que apenas o diuron teve resultados significativos, devido ao maior índice de matéria seca da planta daninha na presença de palha, resultando em baixa eficácia de controle. Ao comparar os três herbicidas na presença de palha, observou-se que apenas tebuthiuron diferenciou-se dos demais, apresentando baixo valor de matéria seca e, portanto, eficaz no controle da planta daninha. Na ausência da palha os herbicidas não diferiram significativamente entre si.

Tabela 6. Valores médios de matéria seca (g) da parte aérea de I. grandifolia aos 30 dias após a aplicação (DAA) para diuron, metribuzin e tebuthiuron com e sem palha (SD = Desvio Padrão). Piracicaba/SP, 2013.

\begin{tabular}{cccc}
\hline \multirow{2}{*}{ Palha } & \multicolumn{3}{c}{ Herbicidas } \\
\cline { 2 - 4 } & Diuron \pm SE & Metribuzin \pm SE & Tebuthiuron \pm SE \\
\hline Com & $0,24 \pm 0,27 \mathrm{Aa}$ & $0,25 \pm 0,36 \mathrm{Aa}$ & $0,05 \pm 0,11 \mathrm{Ab}$ \\
Sem & $0,08 \pm 0,18 \mathrm{Ba}$ & $0,17 \pm 0,38 \mathrm{Aa}$ & $0,08 \pm 0,20 \mathrm{Aa}$ \\
\hline
\end{tabular}

Médias seguidas por letras iguais, maiúsculas nas colunas e minúsculas nas linhas, não diferem entre si pelo teste de Tukey nível de significância de 5\%. 
Tabela 7. Valores médios para avaliação de controle (\%) de Cucumis sativus aos 21 dias após a aplicação (DAA) para diuron, metribuzin e tebuthiuron em diferentes precipitações (SD = Desvio Padrão). Piracicaba/SP, 2013.

\begin{tabular}{cccc}
\hline $\begin{array}{c}\text { Precipitação } \\
\left(\mathbf{m m ~ h a}^{-1}\right)\end{array}$ & Diuron \pm SE & Metribuzin \pm SE & Tebuthiuron \pm SE \\
\cline { 2 - 4 } $\mathbf{0}$ & $46,56 \pm 38,50 \mathrm{Cc}$ & $94,69 \pm 9,74 \mathrm{Aa}$ & $73,13 \pm 26,76 \mathrm{Bb}$ \\
$\mathbf{5}$ & $74,69 \pm 37,17 \mathrm{Bb}$ & $95,31 \pm 9,39 \mathrm{Aa}$ & $90,94 \pm 13,44 \mathrm{Aa}$ \\
$\mathbf{1 0}$ & $93,44 \pm 20,55 \mathrm{Aa}$ & $96,88 \pm 8,54 \mathrm{Aa}$ & $93,75 \pm 11,62 \mathrm{Aa}$ \\
$\mathbf{2 0}$ & $100,00 \pm 0,00 \mathrm{Aa}$ & $100,00 \pm 0,00 \mathrm{Aa}$ & $100,00 \pm 0,00 \mathrm{Aa}$ \\
$\mathbf{2 0 + 2 0}$ & $100,00 \pm 0,00 \mathrm{Aa}$ & $100,00 \pm 0,00 \mathrm{Aa}$ & $99,69 \pm 1,25 \mathrm{Aa}$ \\
Testemunha & $0,00 \pm 0,00 \mathrm{Da}$ & $0,00 \pm 0,00 \mathrm{Ba}$ & $0,00 \pm 0,00 \mathrm{Ca}$ \\
\hline
\end{tabular}

Médias seguidas por letras iguais, maiúsculas nas colunas e minúsculas nas linhas, não diferem entre si pelo teste de

Tukey nível de significância de 5\%.

Tabela 8. Valores médios para avaliação de controle (\%) de Cucumis sativus aos 21 dias após semeadura para diuron, metribuzin e tebuthiuron com e sem palha (SD = Desvio Padrão). Piracicaba/SP, 2013.

\begin{tabular}{cccc}
\hline \multirow{2}{*}{ Palha } & \multicolumn{3}{c}{ Herbicidas } \\
\cline { 2 - 4 } & Diuron \pm SE & Metribuzin \pm SE & Tebuthiuron \pm SE \\
\hline Com & $60,63 \pm 46,29 \mathrm{Bc}$ & $81,5 \pm 37,35 \mathrm{Aa}$ & $74,06 \pm 37,31 \mathrm{Ab}$ \\
Sem & $77,60 \pm 37,67 \mathrm{Aa}$ & $80,73 \pm 37,22 \mathrm{Aa}$ & $78,44 \pm 38,30 \mathrm{Aa}$ \\
\hline
\end{tabular}

Médias seguidas por letras iguais, maiúsculas nas colunas e minúsculas nas linhas, não diferem entre si pelo teste de Tukey nível de significância de 5\%.

O herbicida diuron promoveu bons índices de controle quando submetido a precipitações a partir de $10 \mathrm{~mm}$ (Tabela 7). O metribuzin não promoveu diferença significativa no controle de Cucumis sativus entre as quantidades de precipitações simuladas após a aplicação, o qual se mostrou muito sensível a esse herbicida. Para tebuthiuron verificou-se boas médias de controle, apesar do controle ter sido um pouco comprometido quando não houve precipitação, diferenciandose dos demais regimes pluviométricos.

Analisando-se a influência da palha sobre o desempenho dos herbicidas (Tabela 8), constatou-se que apenas o diuron teve a eficácia de controle de $C$. sativus reduzida pela presença da palha. Ao comparar os herbicidas entre si, observou-se que na presença de palha, metribuzin, seguido de tebuthiuron e diuron promoveram melhor controle de $C$. sativus, enquanto que na ausência de palha os resultados de controle foram semelhante. Em concordância com esses resultados, Monquero et al. (2008) verificaram que a deposição de palha de cana-de-açúcar reduziu significativamente a eficácia de diuron + hexazinone, tendo significativa redução no controle de sorgo e pepino quando aplicado sobre $15 \mathrm{t} \mathrm{ha}^{-1}$ de palha.

As médias de controle obtidas na presença e ausência de palha diferiram significativamente entre si apenas quando não houve simulação de chuva, indicando que a presença da palha e a ausência de umidade reduziram o controle de $C$. sativus pelos herbicidas (Tabela 9). Na presença de palha observou-se diminuição no controle de $C$. sativus apenas nas precipitações 0 e $5 \mathrm{~mm}$, as quais diferiram dos demais tratamentos quando comparados entre si. Na ausência de palha as médias de controle foram significativamente reduzidas apenas na ausência de chuva quando comparada às demais precipitações Ainda assim obteve-se bom resultado de controle de C. sativus, que chegou a média de $83 \%$. 
Tabela 9. Valores médios para avaliação de controle (\%) de Cucumis sativus aos 21 dias após semeadura em diferentes precipitações após o tratamento com e sem palha ( $\mathrm{SD}=$ Desvio Padrão). Piracicaba/SP, 2013.

\begin{tabular}{ccc}
\hline $\begin{array}{c}\text { Precipitação } \\
\left(\text { mm ha }^{-1}\right)\end{array}$ & Com \pm SE & Palha \\
\cline { 2 - 3 } & $59,38 \pm 37,86 \mathrm{Cb}$ & $83,54 \pm 23,80 \mathrm{Ba}$ \\
$\mathbf{0}$ & $81,25 \pm 32,41 \mathrm{Ba}$ & $92,71 \pm 11,03 \mathrm{Aba}$ \\
$\mathbf{1 0}$ & $91,88 \pm 18,64 \mathrm{Aba}$ & $97,50 \pm 7,22 \mathrm{Aa}$ \\
$\mathbf{2 0}$ & $100,00 \pm 0,00 \mathrm{Aa}$ & $100,00 \pm 0,00 \mathrm{Aa}$ \\
$\mathbf{2 0 + 2 0}$ & $100,00 \pm 0,00 \mathrm{Aa}$ & $99,79 \pm 1,02 \mathrm{Aa}$ \\
Testemunha $^{\mathbf{P}}$ & $0,00 \pm 0,00 \mathrm{Da}$ & $0,00 \pm 0,00 \mathrm{Ca}$ \\
\hline
\end{tabular}

Médias seguidas por letras iguais, maiúsculas nas colunas e minúsculas nas linhas, não diferem entre si pelo teste de

Tukey nível de significância de 5\%.

Tabela 10. Valores médios de matéria seca ( $\mathrm{g}$ ) da parte aérea de Cucumis sativus aos 21 dias após semeadura para diuron, metribuzin e tebuthiuron com e sem palha ( $\mathrm{SD}=$ Desvio Padrão). Piracicaba/SP, 2013.

\begin{tabular}{cc}
\hline Precipitação $\left(\mathbf{m m}^{-h^{-1}}\right)$ & Matéria Seca da Parte Aérea(g) \\
\hline $\mathbf{0}$ & $0,05 \pm 0,10 \mathrm{~B}$ \\
$\mathbf{5}$ & $0,00 \pm 0,00 \mathrm{~B}$ \\
$\mathbf{1 0}$ & $0,00 \pm 0,00 \mathrm{~B}$ \\
$\mathbf{2 0}$ & $0,00 \pm 0,00 \mathrm{~B}$ \\
Testemunha & $0,00 \pm 0,00 \mathrm{~B}$ \\
\hline
\end{tabular}

Médias seguidas por letras iguais, não diferem entre si pelo teste de Tukey nível de significância de 5\%.

Os dados de peso de matéria seca $(\mathrm{g})$ foram submetidos ao teste $\mathrm{F}$ e apresentaram diferenças significativas apenas para o fator precipitação, cujas médias foram comparadas utilizando o teste de Tukey a $5 \%$ de probabilidade. Como resultado verificou-se que apenas a testemunha diferenciou-se das demais precipitações, pois apresentou maior quantidade de matéria seca quando comparadas entre si (Tabela 10).

\section{Conclusões}

A eficácia no controle das espécies Ipomoea grandifolia e Cucumis sativus caracterizou-se pelo diuron ter sido menos eficaz nos tratamentos com palha em comparação ao metribuzin e tebuthiuron. A palha afetou de maneira significativa o controle de plantas daninhas pelos herbicidas na ausência de chuva, sendo que uma chuva de 20 $\mathrm{mm}$ foi suficiente para promover excelentes níveis de controle das plantas daninhas.

\section{Referências}

ALAM ASOCIATION LATINOAMERICANA DE MALEZAS. Recomendaciones sobre unificación de los sistemas de evaluación em ensayos de control de malezas. ALAM, v.1, p.35-38, 1974.

\section{ALVES, S.N.R. Efeito residual de herbicidas aplicados em pré-emergência em diferentes condições de restrição hídrica no solo na cultura da cana-de-açúcar. 2010. 127p. Dissertação (Mestrado em Ciências) - Escola Superior de Agricultura "Luiz de Queiroz", Universidade de São Paulo, Piracicaba, 2010.}


CAVENAGHI, A.L. et al. Dinâmica de herbicidas em palhada de cana-de-açúcar. In: CONGRESSO NACIONAL DA SOCIEDADE DOS TÉCNICOS AÇUCAREIROS E ALCOOLEIROS DO BRASIL, 8., 2002, Recife-PE. Anais... Piracicaba: STAB, 2002. p.170-174.

CAVENAGHI, A.L. et al. Dinâmica do herbicida amicarbazone (Dinamic) aplicado sobre palha de cana-de-açúcar (Saccarum officinarum). Planta Daninha, v.25, n.4, p.831-837, 2007.

CORREIA, N.M.; DURIGAN, J.C. Emergência de plantas daninhas em solo coberto com palha de cana-de-açúcar. Planta Daninha, v.22, n.1, p.11-17, 2004.

LAMOREAUX, R.J. et al. Efficacy of dimethenamid, metolachlor and encapsulated alachlor in soil covered with crop residue. Brighton Crop Protection Conference Weeds, v.3, n.3, p.1015-1020, 1993.

MACIEL, C.D.G.; VELINI, E.D. Simulação do caminhamento da água da chuva e herbicidas em palhadas utilizadas em sistemas de plantio direto. Planta Daninha, v.23, n.3, p.471-481, 2005.

MONQUERO, P.A. et al. Eficácia de herbicidas em diferentes quantidades de palha de cana-de-açúcar no controle de Ipomoea grandifolia. Bragantia, v.68, n.2, p.367-372, 2009.

MONQUERO, P.A. et al. Mobilidade e persistência de herbicidas aplicados em préemergência em diferentes solos. Planta Daninha, v.26, n.2, p.411-417, 2008.

NEGRISOLI, E. et al. Associação do herbicida tebuthiuron com a cobertura de palha no controle de plantas daninhas no sistema de cana-crua. Planta Daninha, v.25, n.3, p.621628, 2007.

NEGRISOLI, E. et al. Eficácia do herbicida oxyfluorfen com a cobertura de palha no controle de plantas daninhas. Planta Daninha, v.27, n.1, p.197-203, 2009.

RODRIGUES, B.N.; ALMEIDA, F.S. Guia de herbicidas. 6. ed. Londrina, 2011. 697 p.

ROSSI, C.V.S. et al. Dinâmica do herbicida metribuzin aplicado sobre palha de cana-deaçúcar (Saccharum officinarum). Planta Daninha, v.31, n.1, p.223-230, 2013.

SAS INSTITUTE. SAS/STAT ${ }^{\circledR}$ 9.3: user's guide. $2^{\text {nd }}$ ed. Cary, 2010. Disponível em: <http://support.sas.com/documentation/cdl/en/s tatugmcmc/63125/PDF/default/statugmcmc.pdf >. Acesso em: 20 fev. 2012.

TOFOLI, G.R. et al. Dinâmica do tebuthiuron em palha de cana-de -açúcar. Planta Daninha, v.27, n.4, p.815-821, 2009.

VELINI, E.D. et al. Influência da palha na ocorrência de plantas daninhas e na eficiência de herbicidas. In: FANCELLI, A.L.; DOURADO NETO, D. Milho: estratégias de manejo para alta produtividade. Piracicaba: ESALQ/USP/LPV, 2003. p.198-208. 